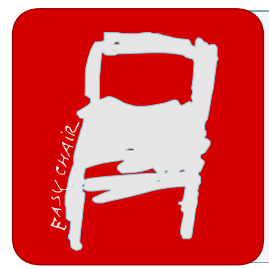

EPiC Series in Health Sciences

CAOS 2017. 17th Annual Meeting of the International Society for Computer Assisted Orthopaedic Surgery

\title{
Ten year survival of navigation-assisted unicompartmental knee arthroplasty
}

\author{
Jean-Yves Jenny \\ University Hospital Strasbourg, Strasbourg, France \\ jean-yves.jennyechru-strasbourg. fr
}

\begin{abstract}
The survival rate of navigation-assisted unicompartmental knee arthroplasty when mechanical revision was considered as the end-point was $94 \%$ after 10 years. These figures compares favorably with previously published literature about conventional implantation. Longer follow-up is required to prove superiority of any technique.
\end{abstract}

\section{Introduction}

Unicompartmental knee arthroplasty (UKA) is considered a highly successful procedure. Survival rates of more than $90 \%$ after 10 years are generally reported. However, complications and revisions may still occur for many reasons, and some of them may be related to the operative technique. Computer assistance has been suggested to improve the accuracy of implantation of a UKA (Jenny 2007). Short term results are encouraging (Jenny 2008, Van der List 2016). However, clinical relevance is still debated (Zhang 2016). The present study was designed to evaluate the long-term (more than 10 years) results of an UKA which was routinely implanted with help of a non-image based navigation system.

The hypothesis of this study will be that the 10 year survival rate of this UKA will be improved in comparison to historical papers when analyzing survival rates and knee function as evaluated by the Knee Society Score (KSS).

\section{Material and methods}

All patients operated on between 2004 and 2005 for implantation of a navigated UKA were eligible for this study. Usual demographic and peri-operative items have been record. All patients were prospectively followed with clinical and radiological examination. All patients were contacted after the 10 year follow-up for repeat clinical and radiological examination (KSS, Oxford knee questionnaire and knee plain X-rays). Patients who did not return were interviewed by phone call. For 
patients lost of follow-up, family or general practitioner was contacted to obtain relevant information about prosthesis survival. Survival curve was plotted according to Kaplan-Meier.

\section{Results}

127 UKAs were implanted during the study time-frame. 117 cases had an optimal lower limb axis (HKA angle between $175^{\circ}$ and $180^{\circ}$ ) after UKA (92\%). Final follow-up (including death or revision) was obtained for 112 cases (88\%). Clinical status after 10 years was obtained for 102 cases (80\%) (KSS, 81 cases - Oxford questionnaire, 96 cases - radiologic evaluation, 74 cases). 9 prosthetic revisions were performed for mechanical reasons during the follow-up time (7\%). The 10 year survival rate was $94 \%$. The mean KSS was 190 points. The mean Oxford score was 56 points. No component was considered loose at the final radiographic evaluation. No polyethylene wear was detected at the final radiographic evaluation.

\section{Discussion}

This study confirms our initial hypothesis, namely quite satisfactory results of a navigated implanted UKA after more than 10 years. Navigation, whose precision is no longer to be demonstrated, probably contributed to the quality of the results. A more consistent anatomical reconstruction and ligamentous balance of the knee should lead to more consistent survival of the UKA. Similar results have already been published (Song 2016). However, superiority of navigated UKA in comparison to conventional implanted UKA is difficult to prove because of the subtle differences expected in mostly underpowered studies. Longer term follow-up may be required.

\section{References}

1. Jenny JY, Ciobanu E, Boeri C, The rationale for navigated minimally invasive unicompartmental knee replacement, Clin Orthop Relat Res, 463, pp: 58-62, 2007.

2. Jenny JY, Unicompartmental knee replacement: a comparison of four techniques combining less invasive approach and navigation, Orthopedics, 31(10 Suppl 1), 2008.

3. Van der List JP, Chawla H, Joskowicz L, Pearle AD, Current state of computer navigation and robotics in unicompartmental and total knee arthroplasty: a systematic review with meta-analysis, Knee Surg Sports Traumatol Arthrosc, 24(11), pp: 3482-3495, 2016.

4. Song EK, N M, Lee SH, Na BR, Seon JK, Comparison of outcome and survival after unicompartmental knee arthroplasty between navigation and conventional techniques with an average 9-year follow-up, J Arthroplasty, 31(2), pp: 395-400, 2016.

5. Zhang Z, Zhu W, Zhu L, Du Y, Superior alignment but no difference in clinical outcome after minimally invasive computer-assisted unicompartmental knee arthroplasty (MICA-UKA), Knee Surg Sports Traumatol Arthrosc, 24(11), pp: 3419-3424, 2016Jenny JY, Miehlke R, Saragaglia D, Geyer R, Mercier N, Schoenahl JY, Thiel B, Single-radius, multidirectional total knee replacement, Knee Surg Sports Traumatol Arthrosc, 21, pp: 2764-2769, 2013. 


\section{Disclosure}

Jean-Yves JENNY receives royalties from Aesculap, is a paid consultant for Exactech, is an unpaid consultant for FH Orthopedics, is member of the board of the CAOS-International Society and of the French Society for Hip and Knee Surgery 